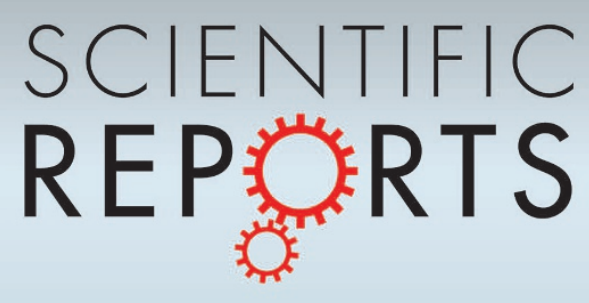

OPEN

SUBJECT AREAS:

MECHANICAL PROPERTIES

THEORY AND COMPUTATION

CONDENSED-MATTER PHYSICS

STRUCTURAL MATERIALS

Received

25 June 2013

Accepted

11 October 2013

Published

29 October 2013

Correspondence and requests for materials should be addressed to

Y.J.T. (fhcl@ysu.edu.

\section{Bulk modulus for polar covalent crystals}

\author{
Bo Xu, Qianqian Wang \& Yongjun Tian
}

State Key Laboratory of Metastable Materials Science and Technology, Yanshan University, Qinhuangdao 066004, China.

A microscopic empirical model of bulk modulus based on atomic-scale parameters is proposed. These parameters include the bond length, the effective bonded valence electron (EBVE) number, and the coordination number product of two bonded atoms, etc. The estimated bulk moduli from our model are in good agreement with experimental values for various polar covalent crystals including ionic crystals. Our current work sheds lights on the nature of bulk modulus, provides useful clues for design of crystals with low compressibility, and is applicable to complex crystals such as minerals of geophysical importance.

$\mathrm{n}$ the past several decades, great efforts have been made to establish direct correlations between the macroscopic mechanical properties and the microscopic parameters of solid. Such correlations can reveal the crucial factors that determine materials mechanical properties. More importantly, they can be used to estimate mechanical properties of designed crystals and provide insightful information before experimental trials. Up to now, some important progresses have been achieved. For example, microscopic hardness models have been constructed in terms of atomic-scale parameters and used extensively experimentally as well as in superhard materials design ${ }^{1}$. However, microscopic models for other mechanical properties, such as bulk modulus ${ }^{2-4}$, are still in the primary stage.

Bulk modulus measures material's resistance to uniform compression, and is highly related to the chemical composition and crystal structure. Materials with high bulk modulus are potentially superhard materials according to the rough correlation between bulk modulus and hardness ${ }^{5}$. Moreover, bulk modulus is relevant to geophysics since it is involved in the interpretation of earth's seismic properties. Numerous experimental and theoretical efforts on bulk modulus have thus been undertaken. However, the measurements of the bulk moduli are nontrivial and values for many materials are still unavailable or unreliable. Concurrently, theoretical calculations of bulk moduli are performed mainly with two approaches. The first is based on ab initio techniques. Unlike hardness, bulk modulus is a strictly defined thermodynamic quantity $(B=-V \mathrm{~d} P / \mathrm{d} V)$, and can thus be obtained theoretically by either using the stain-stress method ${ }^{6}$, or fitting the calculated total energy with Murnaghan equation of state $^{7}$ and Birch-Murnaghan equation of state ${ }^{8}$. These ab initio calculations, relying on computational facilities, can also provide details of the bonding, structural, and electronic properties of solids ${ }^{9}$. The second is empirical approaches based on readily accessible parameters, e.g. chemical bond length, valence, and ionicity. The empirical models can estimate bulk moduli of crystals with the advantage of simplicity and comparable accuracy as $a b$ initio ones, but usually different quantification schemes are being used for different class of crystals.

To put present work in context, a brief review of empirical approaches for bulk modulus estimation is necessary. In 1923, Bridgman proposed an empirical relationship between bulk modulus and molar volume for metals ${ }^{10}$. Sequentially, researchers revealed the bulk moduli of minerals are controlled primarily by the specific volume per ion pair ${ }^{11-13}$. Jayaraman correlated the bulk modulus with the unit cell volume and the effective covalence product for rocksalt-structured rare-earth compounds ${ }^{14}$. It was established that, for a given class of materials with identical crystal structure and similar bonding properties, the bulk modulus would scale with the unit cell volume according to $B=B_{0} V_{0}^{-p}$, where $B_{0}$ and $p$, constants characteristic of selected materials family, are mainly determined by the type of chemical bond (e.g. bond ionicity) and the dependence of the interatomic potential on the interatomic distance, respectively ${ }^{15}$. Hazen and Finger proposed a more general bulk modulusvolume relationship for cation coordination polyhedral in a variety of structure types including oxides, silicates, halides, sulfides, phosphides, and carbides: $B=7.5 S^{2} Z_{a} Z_{c} / \bar{d}^{3}$, where $B, S^{2}, \bar{d}, Z_{a}$ and $Z_{c}$ are the polyhedral bulk modulus, an empirical ionicity, the mean cation-anion separation, cation and anion formal charge, respectively ${ }^{16}$. However, application of this model to rutile-type oxides showed large deviations from experimental values as pointed out by the same authors ${ }^{17}$.

In his seminal work for zincblende semiconductors, Cohen assumed the bulk modulus of covalent crystals should obey the scaling relation between covalent bond energy and the bond volume change, and proposed a bulk modulus model using bond length and an ionicity parameter ${ }^{2}$. The different power-law dependent of bulk 


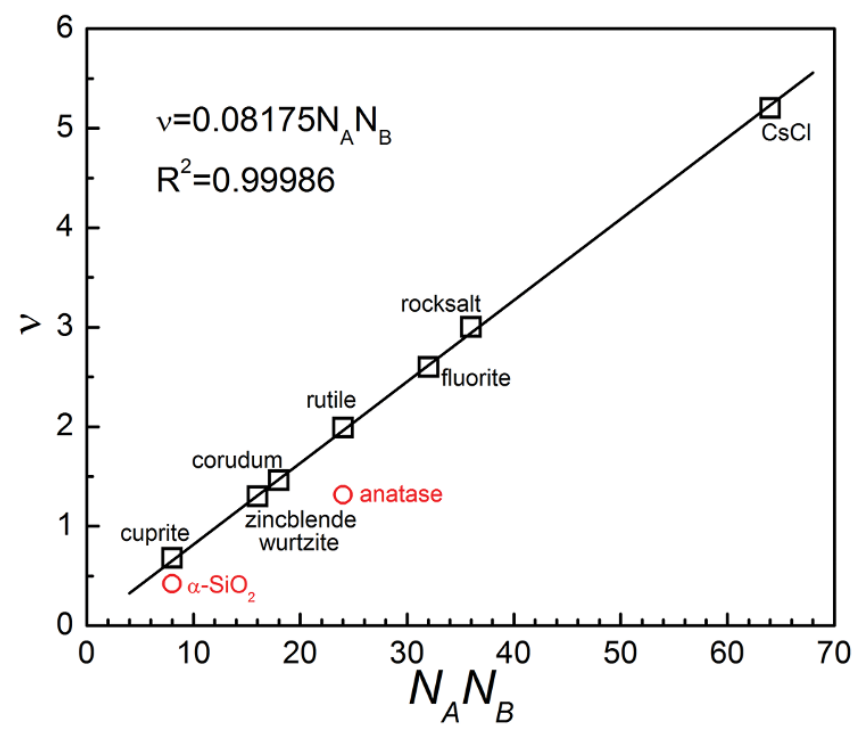

Figure $1 \mid$ Plot of $v$ against the product of coordination numbers of two bonded atoms.

modulus on the bond length for semiconductors $\left(B \propto d^{-3.5}\right)$ and ionic crystals $\left(B \propto d^{-3}\right)$ were also pointed out ${ }^{2}$. Most recently, Cohen's work was developed by Kamran ${ }^{3}$ and Verma ${ }^{18}$ for diamond-like and zincblende covalent crystals. In both cases, the $d^{-3.5}$ dependence of $B$ is retained with different descriptions of the ionicity.

Other empirical bulk modulus models have also been proposed based on different considerations, such as the electronegativity ${ }^{4}$, transition pressure ${ }^{19}$, Debye temperature ${ }^{20}$, plasmon energy ${ }^{21}$, partitioning of the elastic constants with the atomic basin concept ${ }^{22}$, etc. Details of these models are less relevant to present work. It should be noted that some of the key variables used in these models are undeducible from the crystal structural information and have to be provided otherwise, which limit these models' applicability.

The bulk modulus of a crystal microscopically depends on the nature of its chemical bonds such as bond length and type, as corroborated in the empirical relationships between bulk modulus and specific volume (or bond length). Also, a crystal structure dependence of bulk modulus was revealed ${ }^{4,23,24}$. To formulate a microscopic bulk modulus model with easy accessibility and broad applicability, input variables should be directly deducible for selected crystals. Parameterizing of the influences of bond type and crystal structure is thus prerequisite. Recently, we suggested a universal quantification of the bond strength in covalent and ionic crystals by using the proposed effectively bonded valence electron (EBVE) number, $n_{A B}$, of a chemical bond ${ }^{25,26}$, which can be used as a parameter to characterize bond type (See Method section for the details). For singlebond crystals, Levine pointed out the normalized bond volume, $v=$ $n_{b} d^{3} / \Omega$, can be used as a measure of crystal geometry, where $n_{b}, d$, and $\Omega$ are the bond number in the unit cell, bond length, and unit cell volume, respectively ${ }^{27}$. Further analysis shows $v$ is proportional to the product of coordination numbers for two bonded atoms, and distinguishes zincblende, wurtzite, rocksalt, $\mathrm{CsCl}$, cuprite, fluorite, rutile, and corundum structures (Figure 1). This product can thus be used to characterize the crystal structure. We emphasize that both EBVE number and coordination number product can easily be calculated for a designated crystal and applied to multi-bond crystals.

Thus far, three main parameters affecting the bulk modulus, i.e. the bond length $(d)$, EBVE number $\left(n_{A B}\right)$ and coordination number product ( $p=N_{A} N_{B}$ ), are determined. Previous studies revealed the power-law behavior of the bond length (or unit cell volume) on bulk modulus $^{2,15}$. Also, the exponential dependence of the bond strength on EBVE number is emphasized ${ }^{25}$. These studies highlighted the roles of the bond length and valance electrons on bulk modulus, which can be quantified tentatively in terms of fundamental variables with the formalism,

$$
B(\mathrm{GPa})=C p^{l} d^{m} \exp \left(k n_{A B}\right),
$$

where $C, l, m$, and $k$ are the fitting constants. In the following, we deduce the empirical expression of bulk modulus for simple $\mathrm{A}^{\mathrm{N}} \mathrm{B}^{8-\mathrm{N}}$ type covalent crystals with one coordination number. The generalizations to covalent crystals with diverse coordination numbers and to ionic crystals are presented sequentially, followed by generalization to multi-bond crystals. The application of our model to geophysics interesting minerals is also demonstrated.

\section{Results}

$\mathbf{A}^{\mathrm{N}} \mathbf{B}^{8-\mathrm{N}}$ type covalent crystals. We first consider single-bond $\mathrm{A}^{\mathrm{N}} \mathrm{B}^{8-\mathrm{N}}$ type covalent crystals with zincblende, wurtzite, and rocksalt structures where only one type of chemical bond and one coordination number are presented. With the aid of available experimental bulk modulus data (shown in Table 1), our approach is to decompose the bulk modulus into parameters associated with individual chemical bonds, i.e. $d, n_{A B}$, and $p$, by using Eqn. 1 . The constants, $C, l, m$, and $k$, can be extrapolated from the experimental bulk modulus data for these covalent crystals with the LevenbergMarquardt method ${ }^{28,29}$, giving

$$
B(\mathrm{GPa})=54.7 p^{0.914} d^{-3.46} \exp \left(1.485 n_{A B}\right) .
$$

The key variables, experimental bulk moduli ${ }^{3,4,16,30-36}$, and calculated bulk moduli from our model and from other models ${ }^{3,4}$ are listed in Table 1 for selected $\mathrm{A}^{\mathrm{N}} \mathrm{B}^{8-\mathrm{N}}$ type covalent crystals. The comparison between calculated bulk moduli and experimental values is emphasized in Figure 2. The overall accuracy of our model is comparable with the electronegativity $\operatorname{model}^{4}$ and better than that of Kamran et $\mathrm{al}^{3}$. Unlike the other two models, where either electronegativity or ionicity has to be determined through other way, all the input parameters in our model can be directly deduced from the designated crystal with simple arithmetic calculations, providing a unique advantage for its application. To further check the performance of Eqn. 2 for other similar crystals not included in Table 1, we list the results for zincblende-structured $\mathrm{BAs}^{37}$ and $\mathrm{BSb}^{38}$, rocksalt-structured $\mathrm{PbS}^{39}, \mathrm{PbTe}^{40}$, and $\mathrm{AgCl}^{41}$ in Table 2. The consistence of bulk moduli from the experiments ( $a b$ initio calculations for BSb) and our empirical model is satisfying.

$A_{n} B_{m}$ type covalent crystals. For selected $A_{n} B_{m}(n \neq m)$ type crystals (Table 3), bulk moduli calculated from Eqn. 2 are systematically smaller than experimental values ${ }^{13,17,22,42,43}$. It should be noted that an average bond length was used for rutile and corundum structures in Table 2. Previously we chose $p=N_{A} N_{B}$ as a characteristic of crystal geometry, which did not explicitly reveal the diversity of the coordination numbers as presented in $\mathrm{A}_{\mathrm{n}} \mathrm{B}_{\mathrm{m}}$ crystals. Here we define $N_{\max }=\max \left[N_{A}, N_{B}\right]$ and $N_{\min }=\min \left[N_{A}, N_{B}\right]$. We choose two schemes to address this issue. The first one is to use $N_{A}$ and $N_{B}$ individually as input parameters, with the power-law indexes determined by fitting the experimental data. The second one is to introduce an asymmetry parameter, $\Lambda=N_{\max } / N_{\min }$, for two bonded atoms with different coordination numbers. The bulk modulus formulae are $B(\mathrm{GPa})=C N_{\max }^{s} N_{\min }^{t} d^{m} \exp \left(k n_{A B}\right)$ and $B(\mathrm{GPa})=$ $C p^{l} \Lambda^{t} d^{m} \exp \left(k n_{A B}\right)$, respectively. To keep the consistency with Eqn. 2 , only the power-law indexes to $N_{\max }, N_{\min }$, and $\Lambda$ are adjusted during data fitting, and determined to be $1.201,0.672$, and 0.370 , respectively. These two schemes give similar results $\left(B_{\mathrm{cal}, 1}\right.$ and $B_{\mathrm{cal}, 2}$ in Table 3). Here we choose the second scheme,

$$
B(\mathrm{GPa})=54.7 p^{0.914} \Lambda^{0.37} d^{-3.46} \exp \left(1.485 n_{A B}\right) .
$$


Table 1 Parameters related to the calculation of bulk modulus as well as experimental and calculated bulk moduli for simple $A^{N} B^{8-N}$ covalent crystals

\begin{tabular}{|c|c|c|c|c|c|c|c|c|}
\hline Crystal & Stucture & $d$ & $n_{A B}$ & $p$ & $B_{\text {Exp }}$ & $B_{\text {Cal }}$ & $B_{C a l}{ }^{a}$ & $B_{C a l}{ }^{d}$ \\
\hline C & A4 & 1.55 & 0.707 & 16 & $442^{a}$ & 433 & 418.2 & 442 \\
\hline $\mathrm{Si}$ & A4 & 2.35 & 0.707 & 16 & $100^{\circ}$ & 103 & 97.4 & 95.6 \\
\hline $\mathrm{Ge}$ & A4 & 2.45 & 0.707 & 16 & $78^{\circ}$ & 89 & 84.2 & 81.3 \\
\hline Sn & A4 & 2.81 & 0.707 & 16 & $55^{b}$ & 55 & 52.1 & 52.6 \\
\hline iC & B3 & 2.81 & 0.707 & 16 & $211^{a}$ & 222 & 202.9 & 203 \\
\hline $\mathrm{BN}$ & B3 & 1.57 & 0.643 & 16 & $369^{a}$ & 377 & 373 & 398.9 \\
\hline BP & B3 & 1.97 & 0.643 & 16 & $173^{\circ}$ & 172 & 180.4 & 170.8 \\
\hline AlN & B4 & 1.9 & 0.643 & 16 & $201^{a}$ & 195 & 180.9 & 196.6 \\
\hline AlP & B3 & 2.36 & 0.643 & 16 & $86^{\circ}$ & 92 & 88.3 & 89.9 \\
\hline Ass & B3 & 2.43 & 0.643 & 16 & $77^{a}$ & 83 & 80.5 & 79.1 \\
\hline $\mathrm{ISb}$ & B3 & 2.66 & 0.643 & 16 & $59^{\circ}$ & 61 & 56.1 & 61.3 \\
\hline 1 & $\mathrm{P}$ & 1.97 & 0.643 & 16 & $190^{\circ}$ & 172 & 164 & 193.8 \\
\hline GaP & $\mathrm{B}^{3}$ & 2.36 & 0.643 & 16 & $89^{\circ}$ & 92 & 86.6 & 88.8 \\
\hline $\mathrm{aA}$ & B & 2.45 & 0.643 & 16 & $76^{\circ}$ & 81 & 77.4 & 78.6 \\
\hline GaS & B3 & 2.65 & 0.643 & 16 & $57^{\circ}$ & 62 & 59.6 & 62.1 \\
\hline $\ln N$ & B & 2.16 & 0.643 & 16 & $137^{a}$ & 125 & 111.1 & 129.4 \\
\hline $\ln P$ & $\mathrm{~B}^{3} 3$ & .54 & 0.643 & 16 & $73^{\circ}$ & 71 & 66.1 & 69.3 \\
\hline $\ln A s$ & B & 2.61 & 0.643 & 16 & $60^{\circ}$ & 65 & 61.2 & 62.8 \\
\hline - & R & 2.81 & 0.643 & 16 & $47^{a}$ & 50 & 47.7 & 51.4 \\
\hline & B4 & 1.64 & 0.474 & 16 & $244^{c}$ & 252 & & \\
\hline $\mathrm{BeS}$ & $B^{\prime}$ & 2.1 & 0.474 & 16 & $105^{\mathrm{d}}$ & 107 & 132.7 & 112.9 \\
\hline $3 e_{3}$ & $\mathrm{~B}^{3}$ & 2.2 & 0.474 & 16 & $92^{\mathrm{d}}$ & 91 & 113.2 & 95.1 \\
\hline $\mathrm{BeTe}$ & $B$ & 2.4 & 0.474 & 16 & $67^{d}$ & 68 & 86.5 & 72.2 \\
\hline $\lg$ & B & 2.1 & 0.316 & 36 & $163^{\mathrm{e}}$ & 178 & & 154.8 \\
\hline MgS & B1 & 2.6 & 0.316 & 36 & $80^{f}$ & 85 & 72.2 & 76.6 \\
\hline $\mathrm{CaO}$ & B1 & 2.4 & 0.316 & 36 & $114^{9}$ & 112 & & 98.8 \\
\hline 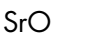 & & $2.5 \varepsilon$ & 0.3 & 36 & $88^{9}$ & 87 & & 78.7 \\
\hline $\mathrm{BaC}$ & B & 2.77 & 0.316 & 36 & $69^{\mathrm{h}}$ & 68 & & 62.5 \\
\hline $\mathrm{ZnO}$ & B & 1.97 & 0.474 & 16 & $139^{d}$ & 134 & & 141 \\
\hline $\mathrm{ZnS}$ & $\mathrm{B}^{3}$ & 2.34 & 0.474 & 16 & $75^{i}$ & 74 & 82.8 & 76.6 \\
\hline $\mathrm{ZnS}$ & $B 3$ & 2.45 & 0.474 & 16 & $62^{\mathrm{d}}$ & 63 & 69.3 & 66.0 \\
\hline $\mathrm{ZnT}$ & B3 & 2.64 & 0.474 & 16 & $51^{d}$ & 49 & 55.6 & 53.4 \\
\hline $\mathrm{Cd}$ & RA & 2.52 & 0.474 & 16 & $62^{\mathrm{d}}$ & 57 & 62.6 & 60.7 \\
\hline $\mathrm{Cds}$ & & 2.62 & 0.4 & 16 & d & 50 & 54.4 & 53.7 \\
\hline $\mathrm{Cd}^{\top}$ & D & 2.81 & 0.4 & 16 & $42^{d}$ & 39 & 42.9 & 44.0 \\
\hline $\mathrm{HeS}$ & $\mathrm{B}^{3}$ & & 0.474 & 16 & $49^{d}$ & 49 & 55.1 & 53.0 \\
\hline $\mathrm{HgT}$ & B3 & 2.78 & 0.474 & 16 & $44^{d}$ & 41 & 46.1 & 44.6 \\
\hline $\mathrm{Mn}$ & B & 2.22 & 0.316 & 36 & $147^{i}$ & 147 & & \\
\hline $\mathrm{FeO}$ & Bl & 2.17 & 0.316 & 36 & $174 i$ & 159 & & \\
\hline
\end{tabular}

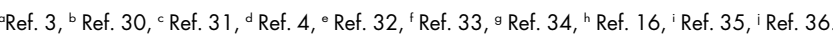

Thus far, a uniform empirical bulk modulus model for both $\mathrm{A}^{\mathrm{N}} \mathrm{B}^{8-\mathrm{N}}$ type and $A_{n} B_{m}$ type covalent crystals is formulated.

Ionic crystals. In examining the bulk moduli for I-VII rocksalt ionic crystals, Cohen gave an approximate scaling, $B=550 d^{-3}$, which differs from that for diamond- and zincblende-structured covalent crystals in the prefactor and power-law index ${ }^{2}$. We use Eqn. 1 to fit the experimental bulk moduli of rocksalt ionic crystals $^{23}$, using fixed $l(0.914)$ and $k(1.485)$. The power-law index $m$ determined here $(-3.43)$ is very close to the previous value $(-3.46)$. For simplicity, an identical value of -3.46 is used, and the empirical bulk modulus formula for rocksaltstructured ionic crystals is deduced as

$$
B(\mathrm{GPa})=25.4 p^{0.914} d^{-3.46} \exp \left(1.485 n_{A B}\right) .
$$

We apply Eqn. 4 to cesium halides with $\mathrm{CsCl}$-structure and produce bulk moduli in great consistent with experimental ones $^{23}$, as listed in Table 4.

Now we consider ionic crystals, such as $\mathrm{Li}_{2} \mathrm{O}, \mathrm{CaF}_{2}, \mathrm{SrF}_{2}$, and $\mathrm{BaF}_{2}$, which possess one type of chemical bond and two distinct coordination numbers. At first glance, the bulk modulus for these crystals should be calculated in the same way as for $A_{n} B_{m}$ type covalent crystals by introducing $\Lambda^{0.37}$ term into Eqn. 4 . However, the calculated bulk moduli are systematically smaller than experimental values except for $\mathrm{Li}_{2} \mathrm{O}^{22,44}$. The ratios of $B_{\text {cal }}$ to $B_{\text {exp }}$ for $\mathrm{CaF}_{2}, \mathrm{SrF}_{2}$, and $\mathrm{BaF}_{2}$ are approximately the same, indicating a common factor we may miss in the bulk modulus formula for ionic crystals. We note an obvious difference in cationic charge state of $\mathrm{Li}(+1)$ and alkaliearth metal atoms $(+2)$ in these crystals. The valence electrons in ionic crystals, unlike those in covalent crystals distributed on the bond, are transferred from cations to anions, which weakens the resistance to compression and results in smaller bulk modulus. It is plausible that cations with higher charge state have a stronger tendency to pull the valence electrons back to the bond region, thus improve the resistibility of chemical bonds to compression and increase the bulk modulus. Therefore, we introduce an additional term, $Q^{\prime}$, to Eqn. 4 to account for such effect, where $Q$ is the cationic charge state and $r$ is the power-law index determined to be 0.58 from $\mathrm{CaF}_{2}, \mathrm{SrF}_{2}$, and $\mathrm{BaF}_{2}$ data. The bulk modulus formula for ionic crystals can be defined as

$$
B(\mathrm{GPa})=25.4 p^{0.914} d^{-3.46} \Lambda^{0.37} Q^{0.58} \exp \left(1.485 n_{A B}\right) .
$$

The key parameters, experimental and calculated bulk moduli are listed in Table 4 for selected ionic crystals. Again, a great consistency is achieved between the calculated bulk moduli and the experimental ones.

Multi-bond crystals. Up to now, we have formulated the bulk modulus for simple polar covalent crystals with parameters deduced from the crystal structure. The generalization of our bulk modulus model to complex multi-bond crystals is straightforward, since all the parameters used in our model are directly correlated to the chemical bond and can easily be applied to complex multi-bond crystals. The only issue is how to weight the contributions to bulk modulus from individual constitutional chemical bonds. Bulk modulus for a multicomponent material can be attributed to the contributions from individual components. From the definition of bulk modulus, $B=-V \mathrm{~d} P / \mathrm{d} V$, we get

$$
\begin{aligned}
\frac{1}{B} & =-\frac{\mathrm{d} V}{\operatorname{Vd} P}=-\frac{\sum_{\mu} \mathrm{d} V_{\mu}}{\mathrm{Vd} P}=\frac{\sum_{\mu} V_{\mu}\left(-d V_{\mu} / \mathrm{V}_{\mu} \mathrm{d} P\right)}{V} \\
& =\frac{\sum_{\mu} V_{\mu} / B_{\mu}}{V}=\sum_{\mu} v_{\mu} / B_{\mu},
\end{aligned}
$$

where $B_{\mu}$ and $v_{\mu}=V_{\mu} / V$ are the bulk modulus and volume fraction of the $\mu$ component, respectively. For a multi-bond crystal, $B_{\mu}$ can be determined by using previous equations, and the volume fraction of distinct chemical bond can be calculated as $v_{\mu}=N_{\mu} d_{\mu}^{3} / \sum_{\mu} N_{\mu} d_{\mu}^{3}$, where $d_{\mu}$ and $N_{\mu}$ are the bond length and bond multiplicity of the $\mu$ type bond in the unit cell, respectively. Bulk modulus for a multibond crystal can then be calculated with Eqn. 6 .

Key variables and bulk moduli are listed in Table 5 for representative multi-bond crystals ${ }^{13,22,45-48}$. The global consistency between calculated bulk modulus and experimentally determined value are remarkably good considering the very simple assessment method and the relatively complex structure in multi-bond crystals. Here we calculate the bulk modulus of spinel $\mathrm{Fe}_{3} \mathrm{O}_{4}$ to show how our model works for multi-bond systems. In $\mathrm{Fe}_{3} \mathrm{O}_{4}$, all oxygen atoms are 4-coordinated, while one third of Fe atoms, Fe1, are 4-coordinated and the other two thirds of $\mathrm{Fe}$ atoms, $\mathrm{Fe} 2$, are 6-coordinated. The bond multiplicities of $\mathrm{O}-\mathrm{Fe} 1$ and $\mathrm{O}-\mathrm{Fe} 2$ bonds are 4 and 12, respectively. $B_{\mu}$ for $\mathrm{O}-\mathrm{Fe} 1$ and $\mathrm{O}-\mathrm{Fe} 2$ components determined from Eqn. 3 are 144 and $188 \mathrm{GPa}$, respectively. Using Eqn. 6, the bulk modulus of spinel $\mathrm{Fe}_{3} \mathrm{O}_{4}$ is calculated to be $177 \mathrm{GPa}$, in good consistent with the experimental value of $187 \mathrm{GPa}^{13}$. Also shown in 


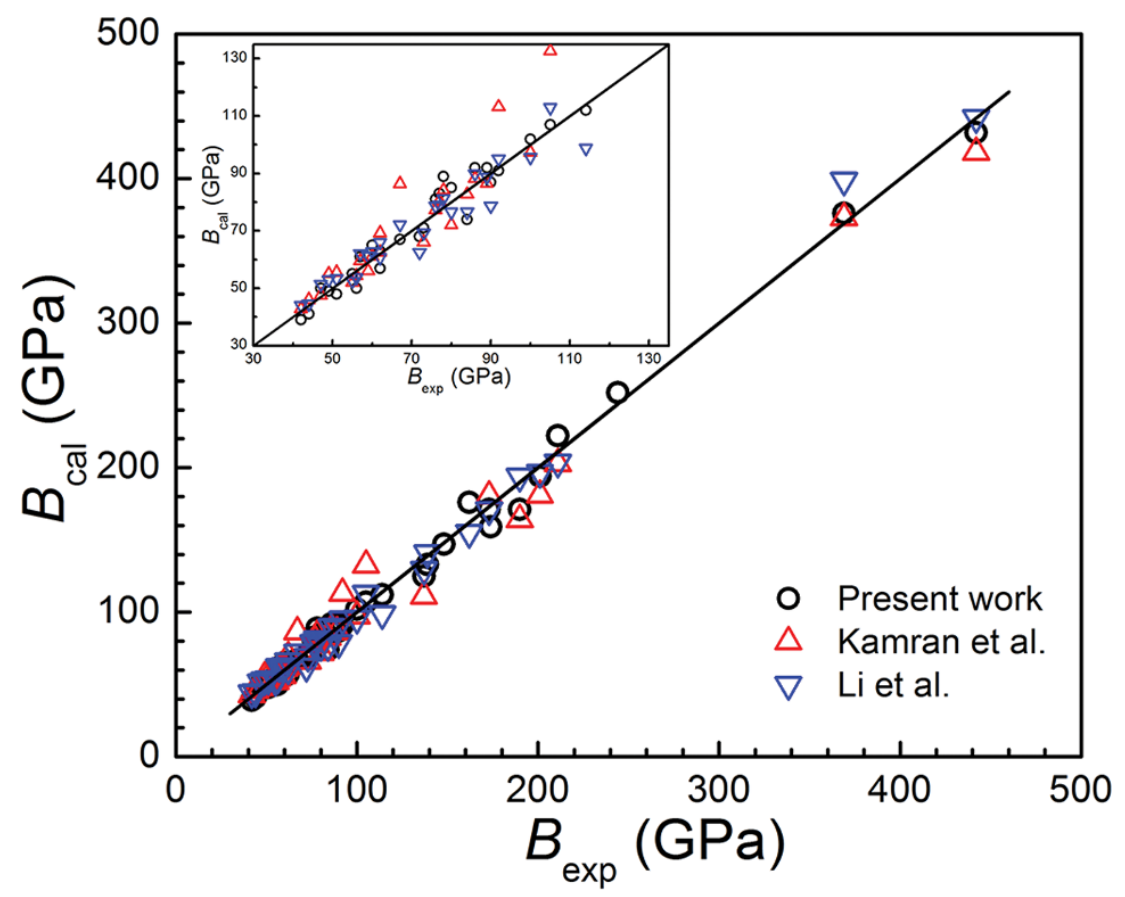

Figure $2 \mid$ Calculated bulk moduli from different models versus the experimental values for simple $\mathrm{A}^{\mathrm{N}} \mathrm{B}^{8-\mathrm{N}}$ type covalent crystals. The inset shows magnified image between 30 and $135 \mathrm{GPa}$.

Table 5 are the theoretically proposed $\beta-\mathrm{C}_{3} \mathrm{~N}_{4}{ }^{46}$ and $\mathrm{z}-\mathrm{BC}_{2} \mathrm{~N}^{47}$ structures. The calculated bulk moduli for these structures with Eqn. 3 and 6 are consistent with the values from first-principles calculations, indicating the prediction power of our simple semi-empirical model.

\section{Discussion}

We can rewrite Eqn. 5 as

$$
B(\mathrm{GPa})=54.7 p^{0.914} d^{-3.46} \Lambda^{0.37} Q_{\text {ionic }} \exp \left(1.485 n_{A B}\right),
$$

where $Q_{\text {ionic }}=0.464 Q^{0.58}$ ( $\mathrm{Q}$ is the cationic charge state) is an ionic term which need to be considered for ionic crystals. We thus formulate our empirical bulk modulus for polar crystals with Eqn. 7 and 6. In Figure 3, bulk moduli from first principles calculations and our empirical model are compared with respect to the experimental values for some $\mathrm{A}^{\mathrm{N}} \mathrm{B}^{8-\mathrm{N}}$ type covalent crystals. Obviously, the accuracy of our model is comparable to LDA-U results and better than LDA-N, GGA-U, and GGA-N results. The advantage of our empirical model is obvious for saving a lot of calculation endeavor compared with these first-principles calculation methods.

It is interesting to further test our model to some geophysical interested materials. These materials usually have complex crystal structures and demonstrate polymorphism, which are difficult to be

Table 2 | Bulk moduli determined from experiments (ab initio calculations for BSb) and Eqn. 2 for zincblende-structured BAs and $\mathrm{BSb}$, rocksalt-structured $\mathrm{PbS}, \mathrm{PbTe}$, and $\mathrm{AgCl}$

\begin{tabular}{|c|c|c|c|c|c|c|}
\hline Crystal & Stucture & $d$ & $n_{A B}$ & $p$ & $B_{\operatorname{Exp}}$ & $B_{\text {Cal }}$ \\
\hline$\overline{B A s}$ & B3 & 2.07 & 0.643 & 16 & $148^{a}$ & 145 \\
\hline $\mathrm{BSb}$ & B3 & 2.22 & 0.643 & 16 & $116^{\mathrm{b}}$ & 114 \\
\hline $\mathrm{PbS}$ & B 1 & 2.97 & 0.316 & 36 & $50^{c}$ & 54 \\
\hline $\mathrm{PbTe}$ & B 1 & 3.23 & 0.316 & 36 & $38^{d}$ & 40 \\
\hline $\mathrm{AgCl}$ & B 1 & 2.89 & 0.165 & 36 & $47^{e}$ & 47 \\
\hline
\end{tabular}

treated even with state of the art calculation methods. In Table 6 we list results for polymorphous $\mathrm{Mg}_{2} \mathrm{SiO}_{4}$, i.e. forsterite $(\alpha)^{49}$, wadsleyite $(\beta)^{50}$, and ringwoodite $(\gamma) \mathrm{Mg}_{2} \mathrm{SiO}_{4}{ }^{51}$, based on our model. These materials are the main constituents of the middle and lower crust as well as upper mantle of the earth. The knowledge of the elastic properties of them is of great importance to understand earth's seismic properties. Our bulk modulus estimations, as listed in Table 6, clearly reflex the relative values of different phases of $\mathrm{Mg}_{2} \mathrm{SiO}_{4}$ with an acceptable accuracy. We compare the bulk moduli of $\alpha-, \beta-$, and $\gamma$ $\mathrm{Mg}_{2} \mathrm{SiO}_{4}$ from first principles calculations and our empirical model with respect to the experimental values (See the inset to Figure 3). Although the empirical model estimates bulk modulus with accuracy inferior to those from first principles calculations, the divergence is in the range of experimental error. Considering the simple formula of our model, it can thus be used to quickly estimate bulk modulus for complex crystals.

Some cautions must be exercised during applying our bulk modulus model. First, three dimensional bond network with clearly defined bonds are needed since our model is based on the partitioning of bulk modulus into individual bonds. Our empirical model thus

Table 3 | Parameters as well as experimental and calculated bulk moduli for $A_{n} B_{m}$ covalent crystals

\begin{tabular}{|c|c|c|c|c|c|c|c|c|}
\hline Crystal & Stucture & $d$ & $n_{A B}$ & $p$ & $\Lambda$ & $B_{\operatorname{Exp}}$ & $B_{C a l}, 1$ & $B_{\text {Cal, } 2}$ \\
\hline $\mathrm{Ag}_{2} \mathrm{O}$ & C3 & 2.044 & 0.474 & 8 & 2 & $84^{a}$ & 79 & 81 \\
\hline $\mathrm{Cu}_{2} \mathrm{O}$ & C3 & 1.85 & 0.474 & 8 & 2 & $112^{a}$ & 111 & 114 \\
\hline $\mathrm{ZrO}_{2}$ & $\mathrm{Cl}$ & 2.195 & 0.474 & 32 & 2 & $235^{\mathrm{b}}$ & 223 & 222 \\
\hline $\mathrm{TiO}_{2}$ & $\mathrm{C} 4$ & 1.96 & 0.596 & 18 & 2 & $224^{c}$ & 233 & 235 \\
\hline $\mathrm{SnO}_{2}$ & C4 & 2.05 & 0.596 & 18 & 2 & $205^{d}$ & 200 & 201 \\
\hline $\mathrm{GeO}_{2}$ & C4 & 1.878 & 0.596 & 18 & 2 & $258^{\mathrm{e}}$ & 269 & 271 \\
\hline $\mathrm{Al}_{2} \mathrm{O}_{3}$ & $\mathrm{D}_{1}$ & 1.915 & 0.474 & 24 & 1.5 & $252^{c}$ & 253 & 246 \\
\hline $\mathrm{Fe}_{2} \mathrm{O}_{3}$ & D5 & 2.025 & 0.474 & 24 & 1.5 & $207^{c}$ & 209 & 203 \\
\hline
\end{tabular}


Table 4 | Parameters as well as experimental and calculated bulk moduli for ionic crystals

\begin{tabular}{|c|c|c|c|c|c|c|}
\hline Crystal & Stucture & $d$ & $n_{A B}$ & $p$ & $B_{\operatorname{Exp}}$ & $B_{\text {Cal }}$ \\
\hline LiF & B1 & 2.01 & 0.165 & 36 & $76.4^{a}$ & 76.7 \\
\hline $\mathrm{LiCl}$ & B1 & 2.57 & 0.165 & 36 & $32.9^{a}$ & 32.8 \\
\hline $\mathrm{LiBr}$ & BI & 2.75 & 0.165 & 36 & $26.0^{a}$ & 25.9 \\
\hline Lil & BI & 3.01 & 0.165 & 36 & $19.3^{a}$ & 19.0 \\
\hline $\mathrm{NaF}$ & BI & 2.31 & 0.165 & 36 & $47.1^{\mathrm{a}}$ & 47.4 \\
\hline $\mathrm{NaCl}$ & Bl & 2.82 & 0.165 & 36 & $23.9^{a}$ & 23.8 \\
\hline $\mathrm{NaBr}$ & BI & 2.99 & 0.165 & 36 & $19.6^{a}$ & 19.4 \\
\hline $\mathrm{Nal}$ & B1 & 3.24 & 0.165 & 36 & $14.9^{a}$ & 14.7 \\
\hline KF & BI & 2.67 & 0.165 & 36 & $28.7^{a}$ & 28.7 \\
\hline $\mathrm{KCl}$ & B1 & 3.15 & 0.165 & 36 & $16.5^{a}$ & 16.2 \\
\hline $\mathrm{KBr}$ & Bl & 3.3 & 0.165 & 36 & $13.9^{a}$ & 13.8 \\
\hline $\mathrm{KI}$ & Bl & 3.53 & 0.165 & 36 & $11.1^{\mathrm{a}}$ & 10.9 \\
\hline $\mathrm{RbF}$ & Bl & 2.83 & 0.165 & 36 & $24.1^{a}$ & 23.5 \\
\hline $\mathrm{RbCl}$ & Bl & 3.29 & 0.165 & 36 & $14.0^{a}$ & 13.9 \\
\hline $\mathrm{RbBr}$ & Bl & 3.44 & 0.165 & 36 & $12.0^{a}$ & 12.0 \\
\hline $\mathrm{Rbl}$ & B1 & 3.67 & 0.165 & 36 & $9.6^{a}$ & 9.6 \\
\hline $\mathrm{CsF}$ & B1 & 3.00 & 0.165 & 36 & $19.3^{a}$ & 19.2 \\
\hline $\mathrm{CsCl}$ & B2 & 3.57 & 0.123 & 64 & $17.6^{a}$ & 16.7 \\
\hline $\mathrm{CsBr}$ & B2 & 3.72 & 0.123 & 64 & $15.4^{\mathrm{a}}$ & 14.5 \\
\hline Csl & B2 & 3.96 & 0.123 & 64 & $12.5^{\mathrm{a}}$ & 11.7 \\
\hline $\mathrm{Li}_{2} \mathrm{O}$ & $\mathrm{Cl}$ & 2.01 & 0.237 & 32 & $88^{b}$ & 100 \\
\hline $\mathrm{CaF}_{2}$ & $\mathrm{Cl}$ & 2.365 & 0.247 & 32 & $84.0^{c}$ & 85.6 \\
\hline $\mathrm{SrF}_{2}$ & $\mathrm{Cl}$ & 2.5 & 0.247 & 32 & $71.3^{c}$ & 70.7 \\
\hline $\mathrm{BaF}_{2}$ & $\mathrm{Cl}$ & 2.683 & 0.247 & 32 & $55.5^{c}$ & 55.3 \\
\hline
\end{tabular}

does not work for transition metal (TM) borides where the chemical bonds between TM atoms and boron atoms are not well defined. Second, our model cannot be applied to crystals such as $\alpha-\mathrm{SiO}_{2}$ and anatase $\mathrm{TiO}_{2}$ with an open-packed crystal structure. In these structures, bonds may not deform iso-structurally under compression: bond bending and/or non-centrosymmetric forces play important roles $^{13}$. These structures can be discriminated by the normalized bond volume $(v)$. As shown in Figure 1, $v$ for $\alpha-\mathrm{SiO}_{2}$ and anatase $\mathrm{TiO}_{2}$ are obviously shifted away from the fitting straight line, indicating an open-packed crystal structure. Third, we note substantial deviations between the calculated bulk moduli from our model and experimental values for TM carbides and nitrides. Unlike TM oxides which are dominated with ionic character, TM carbides and nitrides are characterized with covalent bonds. The difficulty to make an effective assessment for TM carbides and nitrides mainly arises from this covalent character, with additional complexity from the diverse experimental values reported even for the same material. It is well known that the bond strength of $s$ - $p$ - $d$ hybridized chemical bond is greater than that of $s-p$ hybridized chemical bond ${ }^{52}$. In addition, $f$ electrons would push out the spatial extent of the valence $d$-orbitals, allow $d$-electrons to make stronger directional bonds, and enhance bulk modulus ${ }^{53}$. Both effects are not easy to quantify within our simple model. Finally, it should be noted that our microscopic model is deduced based on the experimental bulk modulus data obtained at room temperature. Accordingly, our model works at room temperature and the temperature effect on bulk modulus is out of the scope of current work ${ }^{54,55}$.

To end this paper, we emphasize that our model only need input variables which can be directly deduced from the crystal structure, providing us a general yet powerful tool for bulk modulus evaluation. Pivotal parameters determining bulk modulus are clearly revealed from our model: Short bond length, high EBVE number, and large coordination number are preferred for achieving high bulk modulus. Moreover, the contributions from individual chemical bonds to bulk modulus can readily be partitioned in our model, which is important for design of materials with low compressibility.

\section{Methods}

During compression, it is the disturbed valence electrons that determine the bulk modulus. To establish an effective quantification model, we must find a practical way to estimate the population of these electrons. Considering two atoms, $A$ and $B$, forming a bond in a crystal, the valence electrons are $Z_{A}$ and $Z_{B}$ with coordination numbers of $N_{A}$ and $N_{B}$, respectively. The EBVE number, $n_{A B}$, of $A-B$ bond in terms of $n_{A}$ and $n_{B}$ as

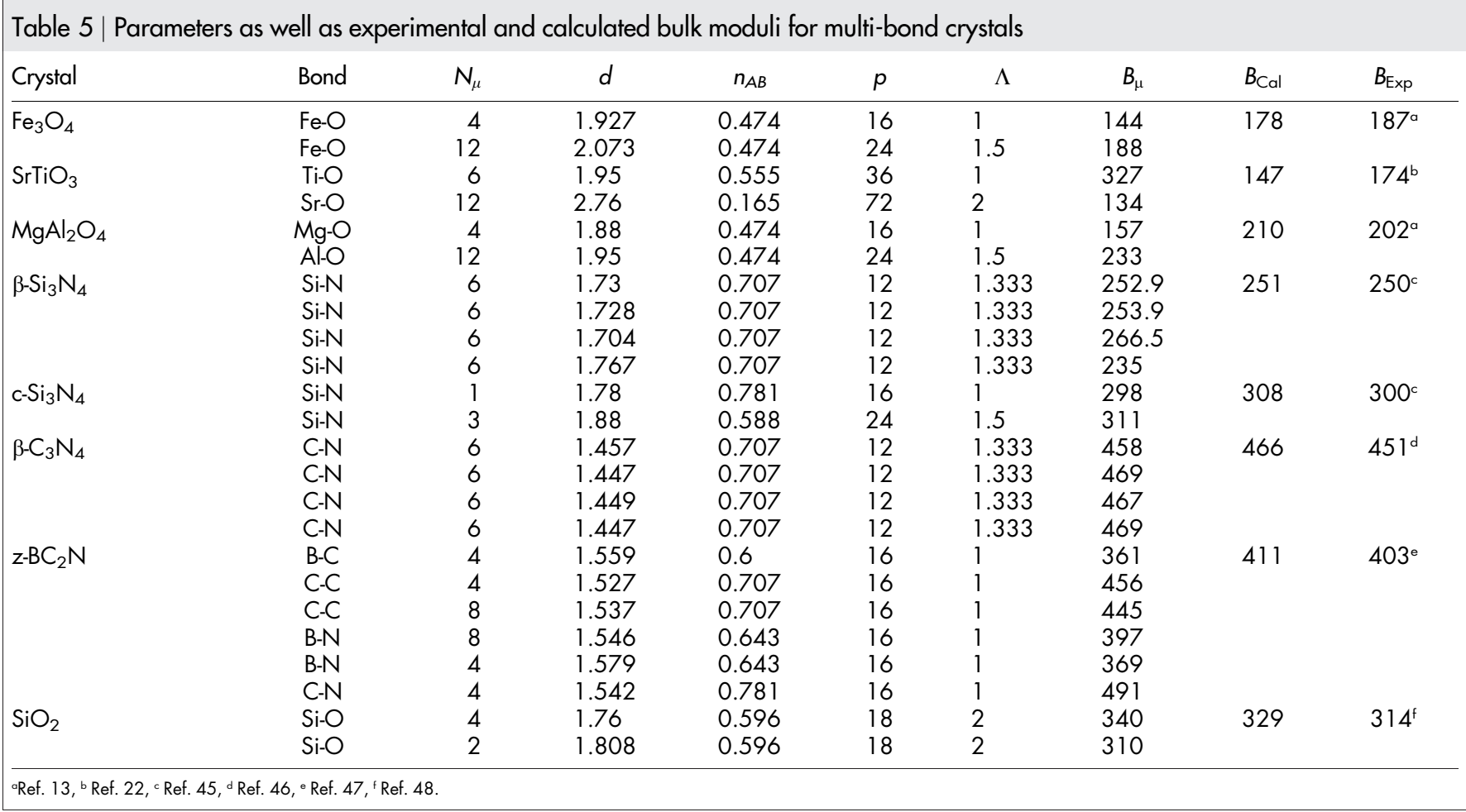




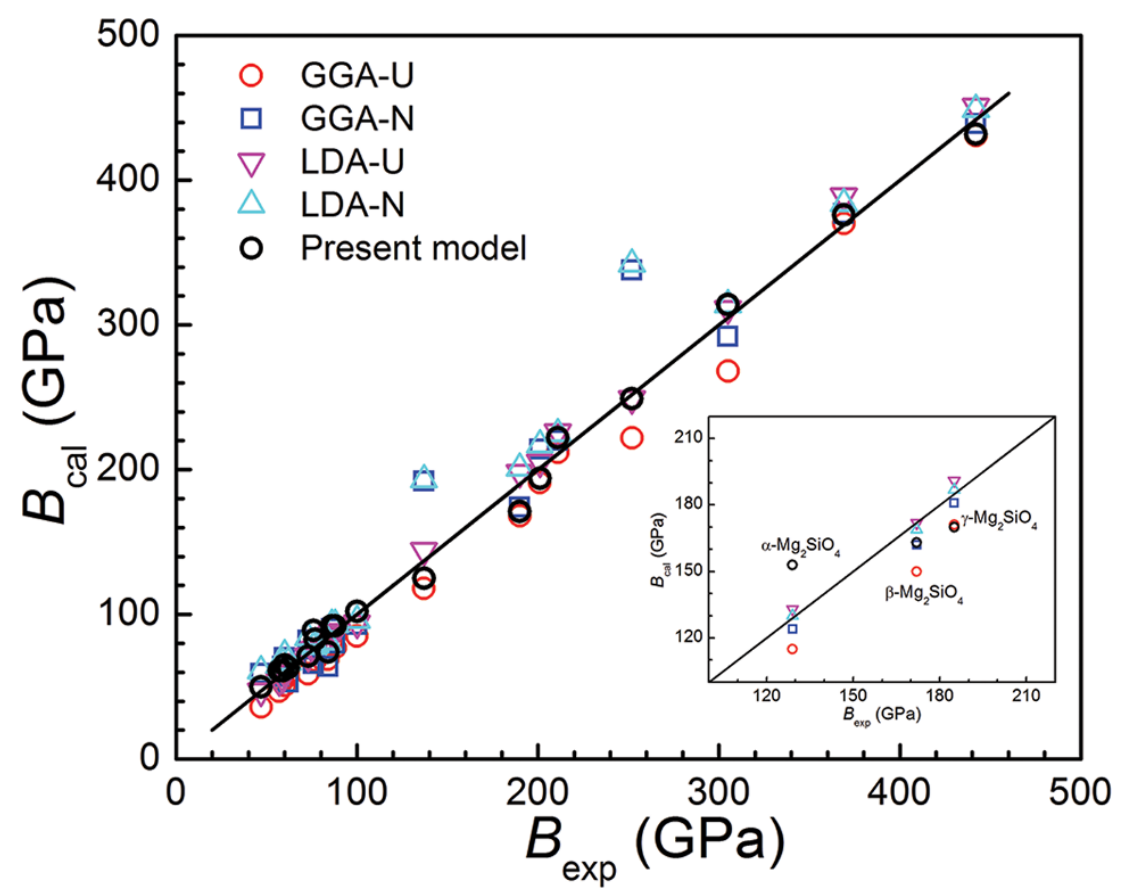

Figure 3 Comparison of bulk moduli deduced from first-principles calculations and our empirical models with respect to the experimental values. The inset shows the corresponding results of $\alpha-, \beta-$, and $\gamma-\mathrm{Mg}_{2} \mathrm{SiO}_{4}$.

$$
n_{A B}=\frac{n_{A} n_{B}}{\sqrt{n_{A}^{2}+n_{B}^{2}}},
$$

where $n_{A}=Z_{A} / N_{A}$ and $n_{B}=Z_{B} / N_{B}$ are the nominal valence electrons contributed to $A-B$ bond. The EBVE numbers of diamond (0.707) and $\mathrm{NaCl}(0.163)$ are in good consistent with the Mulliken overlap population from first-principles calculations. It should be pointed out that lone pair electrons must be excluded during calculations of the nominal valence electrons contributed to $A-B$ bond. For example, in rutile $\mathrm{TiO}_{2}$, oxygen atom is 3 -coordinated with two electrons forming a lone pair (not participating bonding). $n_{\mathrm{o}}$ is thus $4 / 3$ instead of 2 .

For the first-principles calculations, crystal structural relaxations and property calculations were performed using density functional theory within both generalized gradient approximation (GGA) and local density approximation (LDA) as implemented in the CASTEP code ${ }^{56}$. Both ultrasoft (U) and normconserving $(\mathrm{N})$ pseudopotentials were employed in the calculations. $k$-point samplings in the Brillouin zone were chosen by using the Monkhorst-Pack scheme with a resolution of $2 \pi \times 0.04 \AA$. The elastic constants, $C_{\mathrm{ij}}$, were calculated theoretically based on the stress and strain relation (Hooke's law), then the bulk moduli for polycrystals were estimated using the Voigt-Reuss-Hill approximation which is the arithmetic average of the upper (Voigt) and lower (Reuss) bounds for the actual macroscopic effective elastic constants ${ }^{6}$. The calculated bulk moduli are labeled with GGA-U, GGA-N, LDA-U, and LDA-N according to the selected electron-electron exchange functional and pseudopotential.

\begin{tabular}{|c|c|c|c|c|c|c|c|c|c|}
\hline Crystal & Bond & $N_{\mu}$ & $d$ & $n_{A B}$ & $p$ & $\Lambda$ & $B_{\mu}$ & $B_{\text {Cal }}$ & $B_{\mathrm{Exp}}$ \\
\hline \multirow{10}{*}{$\alpha-\mathrm{Mg}_{2} \mathrm{SiO}_{4}$} & $\mathrm{Mg}-\mathrm{O}$ & 16 & 2.131 & 0.325 & 24 & 1.5 & 137 & 153 & $129^{\circ}$ \\
\hline & $\mathrm{Mg}-\mathrm{O}$ & 16 & 2.084 & 0.325 & 24 & 1.5 & 148 & & \\
\hline & $\mathrm{Mg}-\mathrm{O}$ & 16 & 2.068 & 0.325 & 24 & 1.5 & 152 & & \\
\hline & Mg-O & 16 & 2.065 & 0.325 & 24 & 1.5 & 152 & & \\
\hline & $\mathrm{Mg}-\mathrm{O}$ & 16 & 2.21 & 0.325 & 24 & 1.5 & 121 & & \\
\hline & $\mathrm{Mg}-\mathrm{O}$ & 8 & 2.177 & 0.325 & 24 & 1.5 & 127 & & \\
\hline & $\mathrm{Mg}-\mathrm{O}$ & 8 & 2.045 & 0.325 & 24 & 1.5 & 158 & & \\
\hline & Si-O & 16 & 1.637 & 0.832 & 16 & 1 & 431 & & \\
\hline & Si-O & 8 & 1.613 & 0.832 & 16 & 1 & 453 & & \\
\hline & Si-O & 8 & 1.655 & 0.832 & 16 & 1 & 415 & & \\
\hline \multirow{11}{*}{$\beta-\mathrm{Mg}_{2} \mathrm{SiO}_{4}$} & $\mathrm{Mg}-\mathrm{O}$ & 16 & 2.046 & 0.325 & 24 & 1.5 & 158 & 163 & $172^{b}$ \\
\hline & $\mathrm{Mg}-\mathrm{O}$ & 8 & 2.115 & 0.325 & 24 & 1.5 & 141 & & \\
\hline & $\mathrm{Mg}-\mathrm{O}$ & 16 & 2.093 & 0.325 & 24 & 1.5 & 146 & & \\
\hline & $\mathrm{Mg}-\mathrm{O}$ & 4 & 2.095 & 0.325 & 24 & 1.5 & 146 & & \\
\hline & $\mathrm{Mg}-\mathrm{O}$ & 4 & 2.035 & 0.325 & 24 & 1.5 & 161 & & \\
\hline & $\mathrm{Mg}-\mathrm{O}$ & 16 & 2.123 & 0.325 & 24 & 1.5 & 139 & & \\
\hline & $\mathrm{Mg}-\mathrm{O}$ & 16 & 2.128 & 0.325 & 24 & 1.5 & 138 & & \\
\hline & $\mathrm{Mg}-\mathrm{O}$ & 16 & 2.016 & 0.325 & 24 & 1.5 & 166 & & \\
\hline & Si-O & 8 & 1.638 & 0.832 & 16 & 1 & 430 & & \\
\hline & Si-O & 16 & 1.632 & 0.832 & 16 & 1 & 436 & & \\
\hline & Si-O & 8 & 1.701 & 0.832 & 16 & 1 & 378 & & \\
\hline \multirow[t]{2}{*}{$\gamma-\mathrm{Mg}_{2} \mathrm{SiO}_{4}$} & $\mathrm{Mg}-\mathrm{O}$ & 96 & 2.066 & 0.325 & 24 & 1.5 & 153 & 168 & $185^{\mathrm{b}}$ \\
\hline & $\mathrm{Si}-\mathrm{O}$ & 32 & 1.665 & 0.832 & 16 & 1 & 407 & & \\
\hline
\end{tabular}


1. Tian, Y., Xu, B. \& Zhao, Z. Microscopic theory of hardness and design of novel superhard crystals. Int. J. Refract. Met. Hard Mater. 33, 93-106 (2012).

2. Cohen, M. L. Calculation of bulk moduli of diamond and zincblende solids. Phys. Rev. B 32, 7988-7991 (1985).

3. Kamran, S., Chen, K. \& Chen, L. Semiempirical formulae for elastic moduli and brittleness of diamondlike and zinc-blende covalent crystals. Phys. Rev. B 77, 094109 (2008).

4. Li, K., Ding, Z. \& Xue, D. Electronegativity-related bulk moduli of crystal materials. Phys. Status Solidi B 248, 1227-1236 (2011).

5. Haines, J., Leger, J. M. \& Bocquillon, G. Synthesis and design of superhard materials. Annu. Rev. Mater. Res. 31, 1-23 (2001).

6. Wu, Z. J. et al. Crystal structures and elastic properties of superhard $\operatorname{IrN}_{2}$ and $\operatorname{IrN}_{3}$ from first principles. Phys. Rev. B 76, 054115 (2007)

7. Murnaghan, F. D. The compressibility of media under extreme pressures. Proc Natl. Acad. Sci. USA 30, 244-247 (1944).

8. Birch, F. Finite elastic strain of cubic crystals. Phys. Rev. 71, 809-824 (1947).

9. Cohen, M. L. The theory of real materials. Annu. Rev. Mater. Sci. 30, 1-26 (2000)

10. Bridgman, P. W. The compressibility of thirty metals as a function of pressure and temperature. Proc. Am. Acad. Arts Sci. 58, 165-242 (1923).

11. Anderson, O. L. \& Nafe, J. E. Bulk modulus-volume relationship for oxide compounds and related geophysical problems. J. Geophys. Res. 70, 3951-3963 (1965).

12. Anderson, D. L. A seismic equation of state. Geophys. J. R. Astro. Soc. 13, 9-30 (1967).

13. Anderson, D. L. \& Anderson, O. L. Bulk modulus-volume relationship for oxides. J. Geophys. Res. 75, 3494-3500 (1970).

14. Jayaraman, A., Batlogg, B., Maines, R. G. \& Bach, H. Effective ionic charge and bulk modulus scaling in rocksalt-structured rare-earth compounds. Phys. Rev. B 26, 3347-3351 (1982).

15. Neumann, H. Bulk modulus-volume-ionicity relationship in tetrahedrally bonded semiconductors. Cryst. Res. Technol. 22, 99-104 (1987).

16. Hazen, R. M. \& Finger, L. W. Bulk modulus-volume relationship for cation-anion polyhedra. J. Geophys. Res. 84, 6723-6728 (1979).

17. Hazen, R. M. \& Finger, L. W. Bulk moduli and high-pressure crystal-structures of rutile-type compounds. J. Phys. Chem. Solids 42, 143-151 (1981).

18. Verma, A. S. Elastic moduli and brittleness of diamondlike and zinc blende structured solids. Mater. Chem. Phys. 135, 106-111 (2012).

19. Al-Douri, Y., Abid, H. \& Aourag, H. Correlation between the bulk modulus and the transition pressure in semiconductors. Mater. Lett. 59, 2032-2034 (2005).

20. Deus, P. \& Schneider, H. A. A simple estimation of the bulk module of ternary chalcopyrite semiconducting compounds by means of the Debye characteristic temperature. Cryst. Res. Technol. 20, 867-869 (1985)

21. Kumar, V., Shrivastava, A. K. \& Jha, V. Bulk modulus and microhardness of tetrahedral semiconductors. J. Phys. Chem. Solids 71, 1513-1520 (2010).

22. Otero-de-la-Roza, A. \& Luaña, V. Topological partition of the elastic constants of crystals. J. Phys. Chem. A 115, 12953-12961 (2011).

23. Narain, S. Analysis of the Debye temperature for $\mathrm{A}^{\mathrm{N}} \mathrm{B}^{8-\mathrm{N}}$ type ionic and partially covalent crystals. Phys. Status Solidi B 182, 273-278 (1994).

24. Verma, A. S. An empirical model for bulk modulus and cohesive energy of rocksalt-, zincblende- and chalcopyrite-structured solids. Phys. Status Solidi B 246, 345-353 (2009)

25. Guo, X. et al. Unbinding force of chemical bonds and tensile strength in strong crystals. J. Phys.: Condens. Matter 21, 485405 (2009).

26. Xu, B., Guo, X. \& Tian, Y. in Graphene simulation (ed Jianru Gong) Ch. 11, 211-226 (InTech, 2011).

27. Levine, B. F. Bond susceptibilities and ionicities in complex crystal-structures. J. Chem. Phys. 59, 1463-1486 (1973).

28. Levenberg, K. A method for the solution of certain non-linear problems in least squares. Q. Appl. Math. 2, 164-168 (1944).

29. Marquardt, D. W. An algorithm for least-squares estimation of nonlinear parameters. SIAM J. Appl. Math. 11, 431-441 (1963).

30. Kamioka, $\mathrm{H}$. Temperature variations of elastic moduli up to eutectic temperature in tin-bismuth alloys. Japan J. Appl. Phys. 22, 1805-1809 (1983).

31. Bentle, G. G. Elastic constants of single-crystal BeO at room temperature. J. Am. Ceram. Soc. 49, 125-128 (1966).

32. Sinogeikin, S. V. \& Bass, J. D. Single-crystal elasticity of $\mathrm{MgO}$ at high pressure. Phys. Rev. B 59, R14141-R14144 (1999).

33. Van Camp, P. E., Van Doren, V. E. \& Martins, J. L. High pressure properties of the alkaline-earth sulphides. Phys. Status Solidi B 190, 193-197 (1995).

34. Bartels, R. A. \& Vetter, V. H. The temperature dependence of the elastic constants of $\mathrm{CaO}$ and SrO. J. Phys. Chem. Solids 33, 1991-1992 (1972).

35. Ves, S., Schwarz, U., Christensen, N. E., Syassen, K. \& Cardona, M. Cubic ZnS under pressure: Optical-absorption edge, phase transition, and calculated equation of state. Phys. Rev. B 42, 9113-9118 (1990).
36. Sirdeshmukh, D. B. \& Subhadra, K. G. Bulk modulus-volume relationship for some crystals with a rock salt structure. J. Appl. Phys. 59, 276 (1986).

37. Greene, R. G., Luo, H., Ruoff, A. L., Trail, S. S. \& DiSalvo, F. J., Jr. Pressure induced metastable amorphization of BAs: Evidence for a kinetically frustrated phase transformation. Phys. Rev. Lett. 73, 2476-2479 (1994).

38. Ferhat, M., Bouhafs, B., Zaoui, A. \& Aourag, H. First-principles study of structural and electronic properties of BSb. J. Phys.: Condens. Matter 10, 7995-8006 (1998).

39. Peresada, G. I., Ponyatovskii, E. G. \& Sokolovskaya, Z. D. Pressure dependence of the elastic constants of PbS. Phys. Status Solidi A 35, K177-K180 (1976).

40. Miller, A. J., Saunders, G. A. \& Yogurtcu, Y. K. Pressure dependences of the elastic constants of PbTe, SnTe and $\mathrm{Ge}_{0.08} \mathrm{Sn}_{0.92}$ Te. J. Phys. C: Solid State Phys. 14, 1569-1584 (1981).

41. Hull, S. \& Keen, D. A. Pressure-induced phase transitions in $\mathrm{AgCl}, \mathrm{AgBr}$, and AgI. Phys. Rev. B 59, 750-761 (1999).

42. Namavar, F. et al. Thermal stability of nanostructurally stabilized zirconium oxide. Nanotechnology 18, 415702 (2007).

43. Haines, J. \& Leger, J. M. X-ray diffraction study of the phase transitions and structural evolution of tin dioxide at high pressure: Relationships between structure types and implications for other rutile-type dioxides. Phys. Rev. B 55, 11144-11154 (1997).

44. White, G. K. Thermal expansion at low temperatures of the alkaline earth fluorides and $\mathrm{PbF}_{2}$. J. Phys. C: Solid State Phys. 13, 4905-4913 (1980).

45. Zerr, A. et al. Synthesis of cubic silicone nitride. Nature 400, 340-342 (1999).

46. Teter, D. M. \& Hemley, R. J. Low-compressibility carbon nitrides. Science 271, 53-55 (1996).

47. Zhou, X.-F. et al. Most likely phase of superhard $\mathrm{BC}_{2} \mathrm{~N}$ by ab initio calculations. Phys. Rev. B 76, 100101 (2007).

48. Ross, N. L., Shu, J. \& Hazen, R. M. High-pressure crystal chemistry of stishovite. Am. Mineral. 75, 739-747 (1990).

49. Isaak, D. G., Anderson, O. L., Goto, T. \& Suzuki, I. Elasticity of single-crystal forsterite measured to 1700 K. J. Geophys. Res.: Solid Earth 94, 5895-5906 (1989).

50. Li, B., Liebermann, R. C. \& Weidner, D. J. Elastic moduli of wadsleyite ( $\beta$ $\mathrm{Mg}_{2} \mathrm{SiO}_{4}$ ) to 7 gigapascals and 873 kelvin. Science 281, 675-677 (1998).

51. Jackson, J. M., Sinogeikin, S. V. \& Bass, J. D. Sound velocities and elastic properties of $\gamma-\mathrm{Mg}_{2} \mathrm{SiO}_{4}$ to $873 \mathrm{~K}$ by Brillouin spectroscopy. Am. Mineral. 85, 296-303 (2000).

52. Pauling, L. The nature of the chemical bond and the structure of molecules and crystals; an introduction to modern structural chemistry. (Cornell University Press, 1960).

53. Grossman, J. C., Mizel, A., Côté, M., Cohen, M. L. \& Louie, S. G. Transition metals and their carbides and nitrides: Trends in electronic and structural properties. Phys. Rev. B 60, 6343-6347 (1999).

54. Garai, J. \& Laugier, A. The temperature dependence of the isothermal bulk modulus at 1 bar pressure. J. Appl. Phys. 101, 023514-023514 (2007).

55. Anderson, O. L. Derivation of Wachtman's equation for the temperature dependence of elastic moduli of oxide compounds. Phys. Rev. 144, 553-557 (1966).

56. Clark, S. J. et al. First principles methods using CASTEP. Z. Kristallogr. 220, 567-570 (2005)

\section{Acknowledgments}

This work was supported by NBRPC (Grant No. 2011CB808205), NSFC (Grants No. 51332005, No. 51121061).

\section{Author contributions}

B.X. and Y.J.T. designed and coordinated the overall study. Q.Q.W. performed the first principles calculations. B.X. and Y.J.T. analyzed all data and wrote the paper. All authors contributed to the discussion of the results.

\section{Additional information}

Competing financial interests: The authors declare no competing financial interests.

How to cite this article: Xu, B., Wang, Q. \& Tian, Y. Bulk modulus for polar covalent crystals. Sci. Rep. 3, 3068; DOI:10.1038/srep03068 (2013).

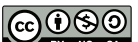

This work is licensed under a Creative Commons AttributionNonCommercial-ShareAlike 3.0 Unported license. To view a copy of this license, visit http://creativecommons.org/licenses/by-nc-sa/3.0 